\title{
The role of small bowel endoscopy in small bowel Crohn's disease: when and how?
}

\author{
Mikang Kim, Hyun Joo Jang \\ Division of Gastroenterology, Department of Internal Medicine, Hallym University Dongtan Sacred Heart Hospital, Hwaseong, Gyeonggi-do, Korea
}

Endoscopy has a crucial role in the diagnosis, management, and surveillance of inflammatory bowel disease (IBD). It contributes in supporting the diagnosis of IBD with the clinical history, physical examination, laboratory findings, and targeted biopsies. Furthermore, endoscopy has a significant role in assessing disease activity and distribution in treatment efficacy evaluation, post-surgical recurrence risk, and cancer surveillance in patients with long-lasting illness. Endoscopy also provides therapeutic potential for the treatment of IBD, especially with stricture dilatation and treatment of bleeding. Small bowel (SB) endoscopy (capsule endoscopy and device-assisted enteroscopy) and cross-sectional radiologic imaging (computed tomography enterography and magnetic resonance enterography) have become important diagnostic options to diagnose and treat patients with SB Crohn's disease. We reviewed the present role of SB endoscopy in patients with SB Crohn's disease. (Intest Res 2016;14:211-217)

Key Words: Crohn disease; Capsule endoscopy; Balloon-assisted enteroscopy

\section{INTRODUCTION}

Crohn's disease (CD) is a chronic IBD that causes gastrointestinal symptoms such as abdominal pain, diarrhea, and bloody stool. CD can affect any part of the gastrointestinal tract, with the involvement of approximately $40 \%$ to $60 \%$ of the small intestine. ${ }^{1,2}$ The terminal ileum is the most common area affected by CD, which is accessible through colonoscopy. ${ }^{3}$ However, the proximal small bowel (SB) is affected in one third of patients with CD with normal ileocolonoscopy. Proximal SBCD can be difficult to diagnose because the proximal SB may not be accessible by conventional colonoscopy.

Chronic IBD is diagnosed by considering all the clinical, endoscopic, imaging, and histologic findings. Endoscopy

Received March 9, 2016. Revised March 9, 2016.

Accepted March 10, 2016.

Correspondence to Hyun Joo Jang, Division of Gastroenterology,

Department of Internal Medicine, Dongtan Sacred Heart Hospital, 7

Keunjaebong-gil, Hwaseong 18450, Gyeonggi-do, Korea. Tel: +82-31-8086-

2450, Fax: +82-31-8086-2029, E-mail: jhj1229@hallym.or.kr

Financial support: None. Conflict of interest: None. plays an essential role in the diagnosis of IBD. ${ }^{4}$ Regarding the role of colonoscopy in the patients with $\mathrm{CD}$, it is important to diagnose accurately, provide differential diagnosis of other inflammatory diseases, and evaluate the extent and activity of diseases. ${ }^{5}$ Furthermore, treatment response can be determined by evaluating the degree of mucosal healing and recurrence of inflammation. ${ }^{5}$ Strictures or bleeding complicated by chronic inflammation can be treated by balloon dilation or endoscopic hemostasis. The role of endoscopy is important for SBCD and colonic CD.

Over the past 10 years, SB examination achieved the advancement of radiologic imaging studies and endoscopic technologies. Traditional SB follow through (SBFT) or SB enteroclysis was recently replaced by CT enterography (CTE) or magnetic resonance enterography (MRE). In the endoscopic field, capsule endoscopy (CE) is a non-invasive luminal evaluation tool for the small intestine using a wireless capsule, which examines the entire SB. Balloon-assisted enteroscopy (BAE) is developed for endoscopic evaluation and therapeutic procedures of the small intestine.

These diagnostic and therapeutic technologies of SB diseases have their strengths and weaknesses. In this article, we

๑ Copyright 2016. Korean Association for the Study of Intestinal Diseases. All rights reserved.

This is an Open Access article distributed under the terms of the Creative Commons Attribution Non-Commercial License (http://creativecommons.org/licenses/by-nc/4.0)

which permits unrestricted non-commercial use, distribution, and reproduction in any medium, provided the original work is properly cited. 
reviewed the literature on the role of endoscopy in SBCD.

\section{Capsule Endoscopy}

CE was introduced in 2002 and revolutionized as small intestine imaging, which provides a noninvasive and sensitive method for complete visualization of the mucosal surface. ${ }^{6}$ Several CE commercial systems exist. They have different technologic features and software specifications: MiroCam, IntroMedic, Seoul, Korea; PillCam SB2, Given Imaging, Yoqneam, Israel; EndoCapsule, Olympus Europe GmbH, Hamburg, Germany; CapsoVision, Saratoga, CA, USA; and OMOM, Chongqing Jinshan Science, Beijing, China. The capsule is passively propelled by intestinal peristalsis while transferring captured images to an external receiver. CE is useful for detection of small mucosal lesions, which are not well detected in radiologic imaging studies. ${ }^{7} \mathrm{CE}$ in patients with CD can identify CD-related mucosal change such as ulcers, erosions, erythema, aphthous lesions, or strictures. ${ }^{8}$

\section{1) Patients With Suspected SBCD}

Retrospective and prospective studies on CE showed that $\mathrm{CE}$ is a useful diagnostic method for the diagnosis of CD in patients with suspected SBCD who have negative colonoscopy results and radiologic imaging tests. A recent metaanalysis reported that diagnostic yields of CE in patients with suspected CD are significantly higher than that of SBFT, SB enteroclysis and CTE (CE, $52 \%$ vs. SBFT/SB enteroclysis, $16 \%$ and incremental yield, $32 \% ; P<0.0001 ; 95 \% \mathrm{CI}$, $16 \%-48 \%$; CE, $68 \%$ vs. CTE, $21 \%$ and incremental yield, $47 \%$; $P<0.00001 ; 95 \% \mathrm{CI}, 31 \%-63 \%){ }^{7}$ Some studies have reported that CE is more useful than MRE for early CD or proximal SB lesion diagnosis. ${ }^{9}$ However, no statistical difference was found in the meta-analysis between CE and MRE for the diagnostic yields of suspected SBCD (CE, 55\% vs. MRE, $45 \%$ and incremental yield, $10 \%$; $95 \% \mathrm{CI}, 14 \%-34 \% ; P=0.43){ }^{7}$

These studies have limitations. The study results may have included false-positive patients because there are no definite criteria for the diagnosis of SBCD of CE, and criteria for suspected SBCD also vary from studies. Several studies showed that CE specificity is low, while its sensitivity is high (Table 1). ${ }^{9-11}$ However, negative predictive values of the CE findings are as high as $97 \%$ in patients with SBCD. ${ }^{12}$ The positive predictive values vary depending on the diagnostic criteria of SBCD in CE and the inclusion criteria of patients with suspected SBCD. ${ }^{12}$ Tukey et al. reported that the positive predictive value of $C E$ was increased in patients selected with the International Conference on Capsule Endoscopy (ICCE) criteria and the presence of more than three ulcers in CE findings, whereas the positive predictive value was only $31 \%$ in patients with any criteria and any lesion among CE findings. ${ }^{12}$ Approximately $14 \%$ of healthy individuals have nonspecific ulcers or erosions in $\mathrm{CE},{ }^{13}$ which could also appear in various $\mathrm{SB}$ diseases such as infection, ischemia, radiation injury, or NSAID-induced enteropathy.

The risk of capsule retention is low (1.6\%) in patients suspected with SBCD without symptoms of obstruction, known stenosis, or history of SB resection. ${ }^{14-16} \mathrm{SB}$ imaging or patency capsule is not routinely recommended before CE in patients with suspected SBCD without obstructive symptoms or known stenosis. ${ }^{14-16}$ Patency capsule or SB imaging such as CTE or MRE before CE is necessary for patients suspected with SBCD with symptoms of obstruction or suspected obstruction of abdominal imaging. ${ }^{17}$ Obtaining careful clinical history is the most important way to avoid the risk of capsule retention before CE.

\section{2) Patients With Known SBCD}

The examination for involvement of SB is necessary to determine the extent and location of inflammation in patients with established CD. Endoscopic investigation is informative in assessing remission maintenance, mucosal healing, or early inflammation recurrence. Furthermore, therapeutic endoscopic procedures could be needed for the diagnosis and treatment of CD complications. Endoscopic investigation with CE can be applied for patients with SBCD and colonic $\mathrm{CD}$ to assess mucosal healing, inflammation recurrence, or identify mucosal inflammation in patients with unexplained abdominal symptoms. The risk of capsule retention is $5 \%$ to $13 \%$ relatively higher in patients with established $\mathrm{CD}^{14,15,18}$

Table 1. Sensitivity and Specificify of Capsule Endoscopy

\begin{tabular}{cccl}
\hline Authors & Sensitivity & Specificity & Indications of patients \\
\hline Solem et al. $(2008)^{10}(n=27)$ & 83 & 53 & Suspected CD \\
Jensen et al. $(2011)^{9}(n=80)$ & 100 & 91 & Suspected and established CD \\
Albert et al. $(2005)^{11}(n=52)$ & 92 & 100 & Suspected and established CD \\
\hline
\end{tabular}

Values are presented as \%. 
SB cross-sectional imaging such as CTE or MRE is usually recommended before CE to identify strictures and assess the transmural and extraintestinal lesions of $\mathrm{CD}{ }^{17}$

Recent meta-analysis showed that CE has significantly higher diagnostic yields than SBFT or CTE in patients with known SBCD (CE, $71 \%$ vs. SBFT/SB enteroclysis, 36\%; incremental yield, 38\%; $P<0.00001 ; 95 \%$ CI, $22 \%-54 \%$; CE, $71 \%$ vs. CTE, 39\%; incremental yield, 32\%; $P \leq 0.0001$; 95\% CI, 16\%$47 \%)^{7}$ On the other hand, diagnostic yields of CE are similar with those of MRE (CE, 70\% vs. MRE, 79\%; incremental yield, $-6 \%$; $P=0.65$; $95 \%$ CI, $-30 \%-19 \%){ }^{7}$ CTE or MRE is useful to identify the acute exacerbation of inflammation in SBCD. ${ }^{19}$ In cases of suspected active inflammation in SB imaging studies without inflammatory activity on colonoscopy, CE can detect inflammatory lesions of the small intestine and can affect therapeutic strategy. ${ }^{20,21}$ Moreover, CE can be helpful for the differential diagnosis between IBS and active CD inflammation, whether abdominal pain or diarrhea is from functional symptoms or active CD.

Mucosal healing is one of the most important end points of long-term prognosis related to the surgery risk or IBD complications. ${ }^{22}$ The remission of clinical symptoms was not well-correlated with endoscopic measures of disease activity. Deep remission, which is defined as endoscopic, biochemical and clinical remission, is an important treatment goal in CD to avoid hospitalization and the need for surgery. A recent study showed that SB inflammation is still detected in most of the patients with $\mathrm{CD}$, who are in clinical and biomarker remission status. SB mucosal healing on CE is achieved in only $15.4 \%$ of patients in clinical remission. ${ }^{20}$ A retrospective study reported that CE findings have clinical impact on therapeutic plans in $52.3 \%$ of patients with established $\mathrm{CD},{ }^{21}$ whereas biomarkers such as CRP or fecal calprotectin are poorly correlated with significant SB inflammation. Therefore, CE findings in patients with established CD may help evaluate the mucosal healing status and response to therapy and can also affect the choice of therapeutic agents. Some studies assess the severity of SB inflammation by using the Lewis score or CECDAI, and further investigations are needed to evaluate these endoscopic scoring indicators. $^{23,24}$

A diagnostic yield of CE has been compared with that of colonoscopy to assess relapse after surgical resection of $\mathrm{CD}$. Some of the studies showed that the diagnostic yield of CE is higher than that of ileocolonoscopy (CE, $62 \%$ vs. ileocolonoscopy, 25\%), ${ }^{25}$ whereas other studies showed that the diagnostic yield of CE is similar with that of ileocolonoscopy (CE, $62 \%-76 \%$ vs. ileocolonoscopy, $61 \%$ ). ${ }^{26} \mathrm{CE}$ is one of the less invasive options in evaluating postoperative recurrence of $\mathrm{CD}$ when colonoscopy is contraindicated or unsuccessful because the anastomosis is not readily accessible.

\section{Balloon-assisted Enteroscopy}

Two types of BAE are available widely. Double-balloon enteroscopy (DBE; Fujinon, Tokyo, Japan) uses two inflatable balloons at each distal end of the enteroscope and overtube, whereas single-balloon enteroscopy (Olympus, Tokyo, Japan) uses only one inflatable balloon at the distal end of overtube.$^{27,28}$ Both BAE provide endoscopic examination of the entire small intestine through antegrade or retrograde approach, biopsies, and therapeutic endoscopic interventions such as balloon dilation or hemostasis. ${ }^{29}$

Endoscopic investigation more readily detects small mucosal lesions such as aphthae, erosions, or small ulcers than radiologic examinations. The diagnostic yields of BAE have been reported in $22 \%-70 \%$ of patients with suspected CD (Fig. 1). ${ }^{30-33}$ The complication rate of diagnostic BAE is approximately $1 \%$, which is relatively low. ${ }^{30,32,33}$ A meta-analysis recently reported that the diagnostic yield of DBE for inflammatory lesions is approximately $16 \%$, which has no significant difference compared with that of CE $(18 \%) \cdot{ }^{34} \mathrm{CE}$ is relatively less invasive and provides information about the insertion route of the DBE. Therefore, CE is recommended as the first test before DBE in patients without fistula or stenosis. ${ }^{34}$

No sufficient data support BAE for the evaluation and management of SBCD. A recent study has compared the activity index of MRE and the simplified endoscopic activity score for CD of single-balloon enteroscopy in known patients with SBCD. This study showed that activity index of magnetic resonance well correlated with the simplified endoscopic activity score for CD in the SB. ${ }^{35}$ Furthermore, the study will be needed on whether radiologic imaging such as MRE or CTE would replace enteroscopic visualization to assess the inflammatory activity of SBCD.

BAE with SB biopsy is necessary for the diagnosis of CD or the exclusion of inflammatory diseases or neoplastic diseases in patients with no confirmative findings of $\mathrm{CD}$ in colonoscopy and suspected SBCD in the radiologic tests or CE. ${ }^{30,31,36-39}$ In addition, several recent studies showed that DBE detects SB lesions in patients with established CD (Table 2). ${ }^{40-42}$ Stepup therapy in $74 \%$ (26/35) of patients based on DBE findings led to clinical remission in $88 \%(23 / 26)$ of patients. ${ }^{40} \mathrm{BAE}$ has an additional clinical impact on the decision of treatment options in patients with established CD with suspected SB activity and no distal disease activity. 
Table 2. Diagnostic and Therapeutic Impact of Balloon-assisted Enteroscopy (BAE) in Known CD with Suspicious Small Bowel (SB) Activity

\begin{tabular}{|c|c|c|c|c|}
\hline Authors & No. of subjects $(n)$ and BAE & SB activitiy & Change in treatment & Clinical improvement \\
\hline Mensink et al. (2009) & $\begin{array}{l}n=40 \\
52 \text { DBEs }\end{array}$ & $60.0 \%(24 / 40)$ & $\begin{array}{l}75 \% \\
\text { Step up }(n=14) \\
\text { Change in biologics }(n=2) \\
\text { Surgery }(n=2)\end{array}$ & $83.0 \%$ \\
\hline Navaneethan et al. $(2014)^{46}$ & $\begin{array}{l}n=43 \\
53 \text { BAEs }\end{array}$ & $53.4 \%(23 / 43)$ & $\begin{array}{l}41.9 \% \\
\text { Step up }(n=7) \\
\text { Change in biologics }(n=4) \\
\text { Surgery }(n=7)\end{array}$ & $54.5 \%$ \\
\hline Mensink et al. $(2010)^{39}$ & $n=50$ & $\begin{array}{l}70.0 \%(35 / 50) \\
46.0 \%(23 / 50) \text { not } \\
\text { assessed by colonoscopy }\end{array}$ & 74\% step up & $88.0 \%$ \\
\hline Rahman et al. $(2015)^{41}$ & $n=38$ & $87.0 \%(33 / 38)$ & $82 \%(31 / 38)$ & \\
\hline
\end{tabular}

$\mathrm{DBE}$, double balloon enterosopy.
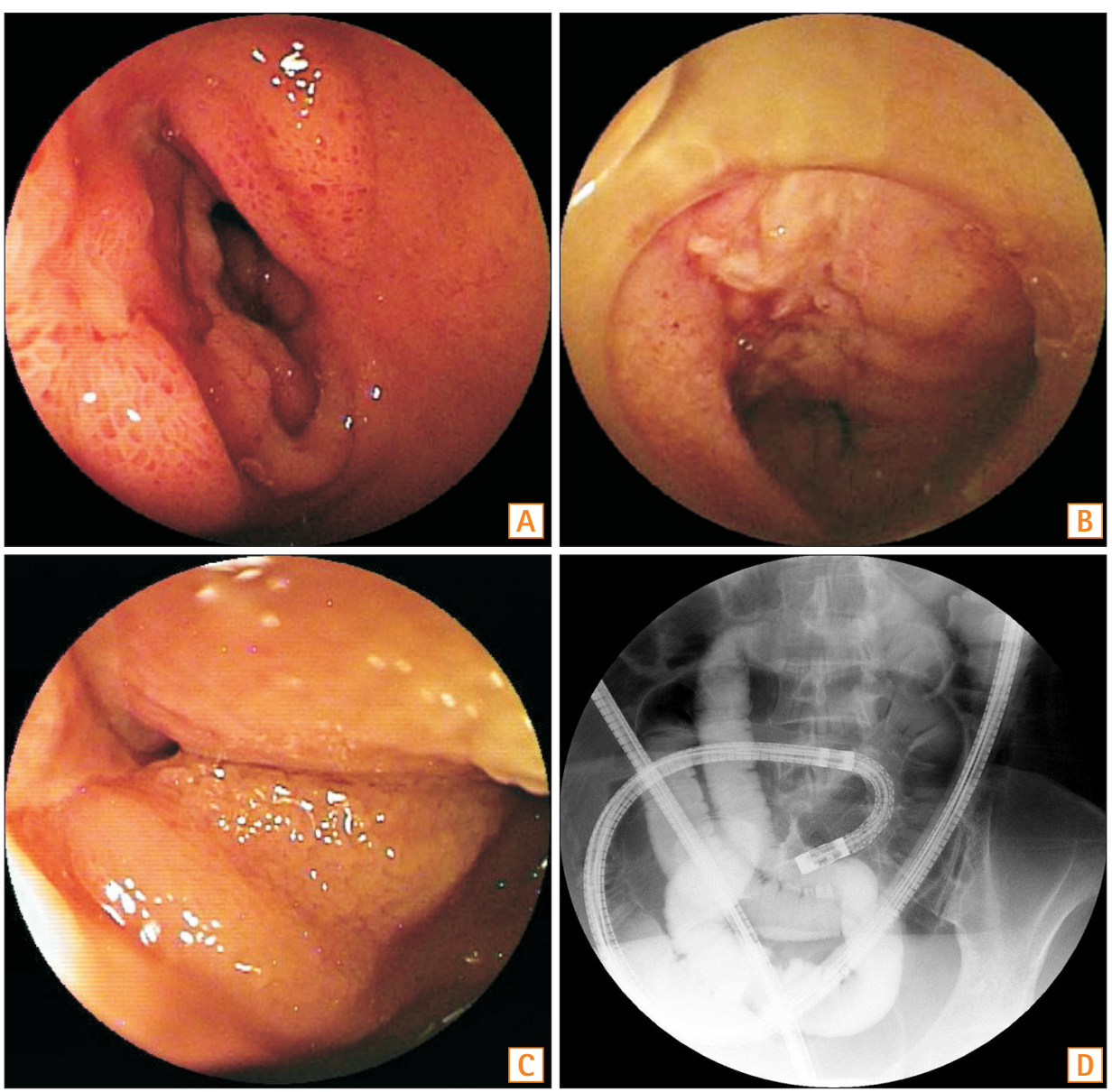

Fig. 1. Enteroscopic findings of small bowel CD. Enteroscopic images of small bowel CD show erosions (A), a longitudinal ulcer (B), and an eccentric ulcer with stricture (C). Enteroscopy is helpful to find a fistulous tract of small intestine through a selective radiography (D).

A recent study reported that 22 patients with suspected SBCD and 43 patients with known SBCD underwent 78 BAEs, and $27.3 \%$ of patients with suspected CD had been diagnosed with CD, and $13.6 \%$, with NSAID enteropathy. ${ }^{43}$ Among patients with known SBCD, active inflammation such as ulcers or stenosis was detected in $41.9 \%$ of patients, which led to a change in therapeutic plans. ${ }^{43} \mathrm{BAE}$ has clinical impact on the diagnosis and treatment of patients with suspected or known SBCD. ${ }^{11}$

BAE provides therapeutic interventions such as hemo- 

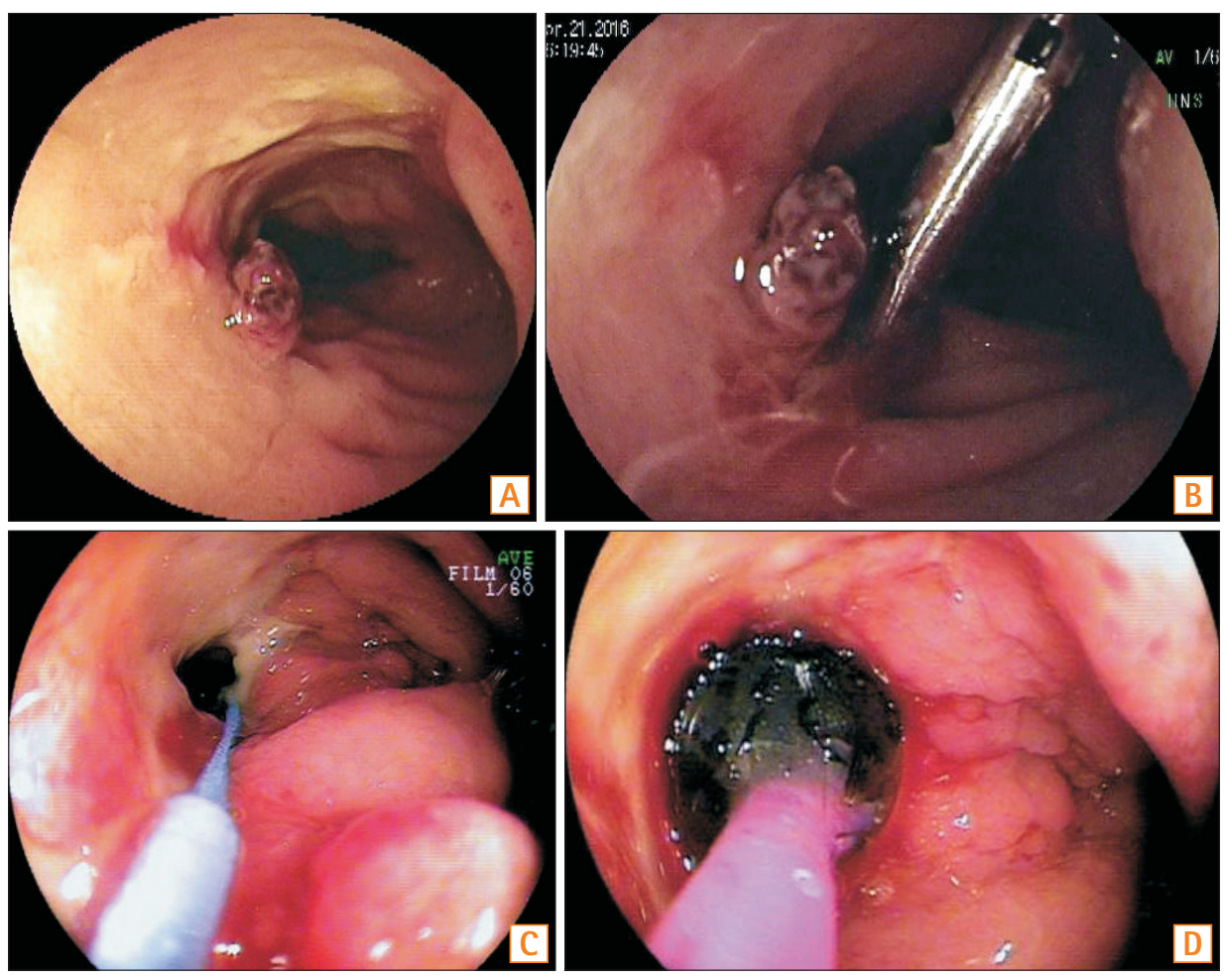

Fig. 2. Therapeutic endoscopy of small bowel $\mathrm{CD}$. Enteroscpic image shows an exposed vessel of deep penetrating ulcers in the patients with small bowel CD presenting massive lower gastrointestinal bleeding (A). Enteroscopy provides hemoclipping on the pulsating vessel (B). There is a severe stricture in the patients with small bowel CD (C). Enteroscopy provides balloon dilation on this short stricture (D).

stasis, balloon dilation, or retrieval of retained capsule in patients with suspected or known CD with bleeding, symptomatic strictures. ${ }^{31,43,44}$ Recent studies showed that technical success of balloon dilation is reported in approximately $60 \%$ to $80 \%$ of patients without deep ulcer or severe inflammation and with strictures less than $5 \mathrm{~cm}$ in length that are endoscopically accessible (Fig. 2).$^{26,30,45}$ However, the risk of perforation following balloon dilation is reported to be $9 \%$, which is higher than the complication rate of diagnostic BAE. ${ }^{30,31,34,40,45,46}$ Balloon dilation should be avoided in strictures longer than $5 \mathrm{~cm}$ or with fistula, severely inflamed, fixed and angulated, or deeply ulcerated stricture.

The advantages of BAE in patients with suspected CD or known CD include the endoscopic evaluation of atypical lesions, obtaining biopsies for histologic diagnosis, and therapeutic potentials such as balloon dilation or hemostasis. However, BAE is technically more complicated and invasive than CE or ileocolonoscopy. Adhesions limit the evaluation of the deeper part of the small intestine because of angulations and strictures, and there are higher risks of complications in patients with active stricturing $\mathrm{CD}$. Therefore, $\mathrm{BAE}$ is recommended in patients with suspected $\mathrm{CD}$ when conventional radiographic studies or colonoscopy has shown inconclusive findings, and histologic evaluation is imperative for management. BAE is also indicated in patients with known CD when endoscopic visualization of the small intestine and biopsies are necessary to differentiate other diseases such as lymphoma, cancer, or tuberculosis, and when therapeutic procedures are essential to treat bleeding, strictures, or retained capsule.

\section{CONCLUSIONS}

SB is the only site involved in around $30 \%$ of patients with $\mathrm{CD}$, although conventional ileocolonoscopy is the first diagnostic method in patients with suspected CD. Therefore, SB evaluation is an important part of the diagnosis of SBCD and continues to evolve because of technologic advances. SB endoscopy (CE or BAE) and cross-sectional imaging (CTE or MRE) have become necessary methods to diagnose and treat patients with CD. CE shows the highest diagnostic yield in patients with suspected CD, but CE has low positive predictive value and modest specificity and should be performed in patients without obstructive symptoms or known stenosis to avoid capsule retention. BAE has advantages in obtaining tissues to differentiate other diseases and therapeutic capabilities, including balloon dilation or hemostasis. On the other hand, CTE and MRE are noninvasive modalities that provide both luminal and extraluminal information. CTE or MRE should be used first in the presence of obstructive symptoms or known strictures. Growing efforts are nowadays made to develop several innovative SB imag- 
ing techniques. These imaging techniques can look beyond the conventional endoscopic techniques, thereby opening a new field to improve the diagnosis and treatment of SBCD, considering the pros and cons of each imaging technique.

\section{REFERENCES}

1. Otterson MF, Lundeen SJ, Spinelli KS, et al. Radiographic underestimation of small bowel stricturing Crohn's disease: a comparison with surgical findings. Surgery 2004;136:854-860.

2. Lescut D, Vanco D, Bonniére P, et al. Perioperative endoscopy of the whole small bowel in Crohn's disease. Gut 1993;34:647-649.

3. Van Assche G, Dignass A, Panes J, et al. The second European evidence-based Consensus on the diagnosis and management of Crohn's disease: definitions and diagnosis. J Crohns Colitis 2010;4:7-27.

4. Leighton JA, Shen B, Baron TH, et al. ASGE guideline: endoscopy in the diagnosis and treatment of inflammatory bowel disease. Gastrointest Endosc 2006;63:558-565.

5. American Society for Gastrointestinal Endoscopy Standards of Practice Committee, Shergill AK, Lightdale JR, et al. The role of endoscopy in inflammatory bowel disease. Gastrointest Endosc 2015;81:1101-1121.

6. Iddan G, Meron G, Glukhovsky A, Swain P. Wireless capsule endoscopy. Nature 2000;405:417.

7. Dionisio PM, Gurudu SR, Leighton JA, et al. Capsule endoscopy has a significantly higher diagnostic yield in patients with suspected and established small-bowel Crohn's disease: a metaanalysis. Am J Gastroenterol 2010;105:1240-1248.

8. Niv Y, Ilani S, Levi Z, et al. Validation of the Capsule Endoscopy Crohn's Disease Activity Index (CECDAI or Niv score): a multicenter prospective study. Endoscopy 2012;44:21-26.

9. Jensen MD, Nathan T, Rafaelsen SR, Kjeldsen J. Diagnostic accuracy of capsule endoscopy for small bowel Crohn's disease is superior to that of MR enterography or CT enterography. Clin Gastroenterol Hepatol 2011;9:124-129.

10. Solem CA, Loftus EV, Jr., Fletcher JG, et al. Small-bowel imaging in Crohn's disease: a prospective, blinded, 4-way comparison trial. Gastrointest Endosc 2008;68:255-266.

11. Albert JG, Martiny F, Krummenerl A, et al. Diagnosis of small bowel Crohn's disease: a prospective comparison of capsule endoscopy with magnetic resonance imaging and fluoroscopic enteroclysis. Gut 2005;54:1721-1727.

12. Tukey M, Pleskow D, Legnani P, Cheifetz AS, Moss AC. The utility of capsule endoscopy in patients with suspected Crohn's disease. Am J Gastroenterol 2009;104:2734-2739.
13. Goldstein JL, Eisen GM, Lewis B, et al. Video capsule endoscopy to prospectively assess small bowel injury with celecoxib, naproxen plus omeprazole, and placebo. Clin Gastroenterol Hepatol 2005;3:133-141.

14. Cave D, Legnani P, de Franchis R, Lewis BS; ICCE. ICCE consensus for capsule retention. Endoscopy 2005;37:1065-1067.

15. Postgate AJ, Burling D, Gupta A, Fitzpatrick A, Fraser C. Safety, reliability and limitations of the given patency capsule in patients at risk of capsule retention: a 3-year technical review. Dig Dis Sci 2008;53:2732-2738.

16. Cheifetz AS, Kornbluth AA, Legnani P, et al. The risk of retention of the capsule endoscope in patients with known or suspected Crohn's disease. Am J Gastroenterol 2006;101:2218-2222.

17. Pennazio M, Spada C, Eliakim R, et al. Small-bowel capsule endoscopy and device-assisted enteroscopy for diagnosis and treatment of small-bowel disorders: European Society of Gastrointestinal Endoscopy (ESGE) Clinical Guideline. Endoscopy 2015;47:352-376.

18. Liao Z, Gao R, Xu C, Li ZS. Indications and detection, completion, and retention rates of small-bowel capsule endoscopy: a systematic review. Gastrointest Endosc 2010;71:280-286.

19. Patel NS, Pola S, Muralimohan R, et al. Outcomes of computed tomography and magnetic resonance enterography in clinical practice of inflammatory bowel disease. Dig Dis Sci 2014;59:838-849.

20. Kopylov U, Yablecovitch D, Lahat A, et al. Detection of small bowel mucosal healing and deep remission in patients with known small bowel Crohn's disease using biomarkers, capsule endoscopy, and imaging. Am J Gastroenterol 2015;110:13161323.

21. Kopylov U, Nemeth A, Koulaouzidis A, et al. Small bowel capsule endoscopy in the management of established Crohn's disease: clinical impact, safety, and correlation with inflammatory biomarkers. Inflamm Bowel Dis 2015;21:93-100.

22. Bharadwaj S, Tandon P, Kulkarni G, Rivas J, Charles R. The role of endoscopy in inflammatory bowel disease. J Dig Dis 2015;16:689-698.

23. Koulaouzidis A, Douglas S, Plevris JN. Lewis score correlates more closely with fecal calprotectin than Capsule Endoscopy Crohn's Disease Activity Index. Dig Dis Sci 2012;57:987-993.

24. Gal E, Geller A, Fraser G, Levi Z, Niv Y. Assessment and validation of the new Capsule Endoscopy Crohn's Disease Activity Index (CECDAI). Dig Dis Sci 2008;53:1933-1937.

25. Pons Beltrán V, Nos P, Bastida G, et al. Evaluation of postsurgical recurrence in Crohn's disease: a new indication for capsule endoscopy? Gastrointest Endosc 2007;66:533-540. 
26. Bourreille A, Jarry M, D'Halluin PN, et al. Wireless capsule endoscopy versus ileocolonoscopy for the diagnosis of postoperative recurrence of Crohn's disease: a prospective study. Gut 2006;55:978-983.

27. Yamamoto H, Sekine Y, Sato Y, et al. Total enteroscopy with a nonsurgical steerable double-balloon method. Gastrointest Endosc 2001;53:216-220.

28. Hartmann D, Eickhoff A, Tamm R, Riemann JF. Balloon-assisted enteroscopy using a single-balloon technique. Endoscopy 2007;39(Suppl 1):E276.

29. May A. Balloon enteroscopy: single- and double-balloon enteroscopy. Gastrointest Endosc Clin N Am 2009;19:349-356.

30. Gay G, Delvaux M. Double balloon enteroscopy in Crohn's disease and related disorders: our experience. Gastrointest Endosc 2007;66(3 Suppl):S82-S90.

31. Manes G, Imbesi V, Ardizzone S, Cassinotti A, Pallotta S, Porro GB. Use of double-balloon enteroscopy in the management of patients with Crohn's disease: feasibility and diagnostic yield in a high-volume centre for inflammatory bowel disease. Surg Endosc 2009;23:2790-2795.

32. Heine GD, Hadithi M, Groenen MJ, Kuipers EJ, Jacobs MA, Mulder CJ. Double-balloon enteroscopy: indications, diagnostic yield, and complications in a series of 275 patients with suspected small-bowel disease. Endoscopy 2006;38:42-48.

33. Jang HJ, Choi MH, Eun CS, et al. Clinical usefulness of double balloon enteroscopy in suspected Crohn's disease: the KASID multi-center trial. Hepatogastroenterology 2014;61:1292-1296.

34. Pasha SF, Leighton JA, Das A, et al. Double-balloon enteroscopy and capsule endoscopy have comparable diagnostic yield in small-bowel disease: a meta-analysis. Clin Gastroenterol Hepatol 2008;6:671-676.

35. Takenaka K, Ohtsuka K, Kitazume Y, et al. Correlation of the endoscopic and magnetic resonance scoring systems in the deep small intestine in Crohn's disease. Inflamm Bowel Dis 2015;21:1832-1838.

36. Sunada K, Yamamoto H, Kita H, et al. Clinical outcomes of enteroscopy using the double-balloon method for strictures of the small intestine. World J Gastroenterol 2005;11:1087-1089.
37. May A, Nachbar L, Ell C. Double-balloon enteroscopy (pushand-pull enteroscopy) of the small bowel: feasibility and diagnostic and therapeutic yield in patients with suspected small bowel disease. Gastrointest Endosc 2005;62:62-70.

38. Prachayakul V, Deesomsak M, Aswakul P, Leelakusolvong S. The utility of single-balloon enteroscopy for the diagnosis and management of small bowel disorders according to their clinical manifestations: a retrospective review. BMC Gastroenterol 2013;13:103. doi: 10.1186/1471-230X-13-103.

39. Mensink PB, Aktas H, Zelinkova Z, West RL, Kuipers EJ, van der Woude CJ. Impact of double-balloon enteroscopy findings on the management of Crohn's disease. Scand J Gastroenterol 2010;45:483-489.

40. Mensink PB, Groenen MJ, van Buuren HR, Kuipers EJ, van der Woude CJ. Double-balloon enteroscopy in Crohn's disease patients suspected of small bowel activity: findings and clinical impact. J Gastroenterol 2009;44:271-276.

41. Rahman A, Ross A, Leighton JA, et al. Double-balloon enteroscopy in Crohn's disease: findings and impact on management in a multicenter retrospective study. Gastrointest Endosc 2015;82:102-107.

42. Oshitani N, Yukawa T, Yamagami H, et al. Evaluation of deep small bowel involvement by double-balloon enteroscopy in Crohn's disease. Am J Gastroenterol 2006;101:1484-1489.

43. Lee BI, Choi H, Choi KY, et al. Retrieval of a retained capsule endoscope by double-balloon enteroscopy. Gastrointest Endosc 2005;62:463-465.

44. Despott EJ, Gupta A, Burling D, et al. Effective dilation of smallbowel strictures by double-balloon enteroscopy in patients with symptomatic Crohn's disease (with video). Gastrointest Endosc 2009;70:1030-1036.

45. Gill RS, Kaffes AJ. Small bowel stricture characterization and outcomes of dilatation by double-balloon enteroscopy: a singlecentre experience. Therap Adv Gastroenterol 2014;7:108-114.

46. Navaneethan U, Vargo JJ, Menon KV, Sanaka MR, Tsai CJ. Impact of balloon-assisted enteroscopy on the diagnosis and management of suspected and established small-bowel Crohn's disease. Endosc Int Open 2014;2:E201-E206. 